KYUNGPOOK Math. J. 50(2010), 89-100

\title{
Weak Hyponomal Composition Operators Induced by a Tree
}

\author{
Mi RyeOng LeE* \\ Faculty of Liberal Education, Kyungpook National University, Daegu 702-701, Ko- \\ rea \\ e-mail : leemr@knu.ac.kr
}

Hyo Gun AHN

Department of Mathematics, College of Natural Sciences, Kyungpook National University, Daegu 702-701, Korea

e-mail : ahk0323@hanmail.net

Abstract. Let $\mathcal{G}=(V, E, \mu)$ be a weighted directed tree, where $V$ is a vertex set, $E$ is an edge set, and $\mu$ is a $\sigma$-finite measure on $V$. The tree $\mathcal{G}$ induces a composition operator $C$ on the Hilbert space $l^{2}(V)$. Hand-type directed trees are defined and characterized the weak hyponormalities of such $C$ in this note. Also some additional related properties are discussed. In addition, some examples related to directed hand-type trees are provided to separate classes of weak-hyponormal operators.

\section{Introduction}

Let $\mathcal{H}$ be a separable, infinite dimensional complex Hilbert space and let $\mathcal{B}(\mathcal{H})$ be the algebra of all bounded linear operators on $\mathcal{H}$. An operator $T$ in $\mathcal{B}(\mathcal{H})$ is said to be normal (or quasinormal) if $T$ commutes with $T^{*}$ (or $T^{*} T$, respectively). An operator $T$ is said to be $p$-hyponormal if $\left(T^{*} T\right)^{p} \geq\left(T T^{*}\right)^{p}$ for $0<p<\infty$. If $p=1$, $T$ is hyponormal, and if $p=\frac{1}{2}, T$ is semi-hyponormal ([11]). For $0<q \leq p<\infty$, every $p$-hyponormal operator is $q$-hyponormal by the Löwner-Heinz inequality $([4])$. For the (unique) polar decomposition of $T=U|T|$ with $\operatorname{ker} U=\operatorname{ker}|T|=\operatorname{ker} T$ and $\operatorname{ker} U^{*}=\operatorname{ker} T^{*}$ and $p>0, T$ is absolute-p-paranormal if $\left\|\left.T\right|^{p} T x\right\| \geq\|T x\|^{p+1}$ for all unit vector $x \in \mathcal{H}$. And $T$ is p-paranormal if $\left\||T|^{p} U|T|^{p} x\right\| \geq\left\||T|^{p} x\right\|^{2}$ for all unit vector $x \in \mathcal{H}$. Note that every $p$-hyponormal operator is absolute- $p$ paranormal, and also absolute- $p$-paranormality is $p$-paranormality for $p>1$. Note that for $0<p<1$, every $p$-hyponormal operator is $p$-paranormal and $p$-paranormal operator is absolute-p-paranormal ([4]).

For recent 30 years, the partial normality has been studied by many operator theorists. In particular, the $p$-hyponormality notion is contained in such studies. Jung-Lee-Park([7]) constructed examples induced by some block matrix operators,

\footnotetext{
* Corresponding Author.
}

Received October 23, 2009; accepted January 25, 2010.

2000 Mathematics Subject Classification: 47B20, 47B33, 94C15.

Key words and phrases: directed tree, composition operator, $p$-hyponormal, $p$-paranormal. 
and in [6], the classes of $p$-hyponormal operators are distinct with respect to positive real numbers $p$. In [1] and [2] Burnap-Jung-Lambert discussed some composition operator models on $L^{2}$, and also they showed that the classes of $p$-hyponormal and $p$-paranormal operators are distinct for each $p>0$. Nonetheless, new examples for the $p$-hyponormal operator classes are needed to study the relationship between the above two notions.

The main tool to study the $p$-hyponormality of a composition operator is the conditional expectations (cf. [2] and [3]), which will be used frequently in this note. Here are some terminology and notation for conditional expectation. Let $(X, \mathcal{F}, \mu)$ be a $\sigma$-finite measure space and let $T: X \rightarrow X$ be a transformation such that $T^{-1} \mathcal{F} \subset \mathcal{F}$ and $\mu \circ T^{-1} \ll \mu$. An operator $C_{T}$ acting on $L^{2}:=L^{2}(X, \mathcal{F}, \mu)$ is defined by $C_{T} f=f \circ T$. The condition of the Radon-Nikodym derivative $h(=d \mu \circ$ $\left.T^{-1} / d \mu\right) \in L^{\infty}$ guarantees that $C_{T}$ is bounded on $L^{2}$. We call $C_{T}$ is a composition operator induce by the transformation $T$. And we denote $E f=E\left(f \mid T^{-1} \mathcal{F}\right)$ for the conditional expectation of $f$ with respect to $T^{-1} \mathcal{F}$. As a special case in [10], if $\mathcal{A}$ is the purely atomic $\sigma$-subalgebra of $\mathcal{F}$ generated by the measurable partition of $X$ into sets of positive measure $\left\{A_{k}\right\}_{k \geq 0}$, then it is well-known that

$$
E(f \mid \mathcal{A})=\sum_{k=0}^{\infty} \frac{1}{\mu\left(A_{k}\right)}\left(\int_{A_{k}} f(x) d \mu(x)\right) \chi_{A_{k}} .
$$

The idea in [2] and [3] provides a good motivation to study composition operator on the Hilbert space $l^{2}(V)$ defined by a vertex set in a weighted directed tree $\mathcal{G}=(V, E, \mu)$ (whose notations are introduced in the next section). For a directed tree $\mathcal{G}$ with masses, a measurable transformation $T$ can be defined on a vertex set $V$. And we consider a bounded composition operator on $l^{2}(V)$ induced by the transformation. In this note, we provide composition operators as a new model which separates various classes of quasinormal, $p$-hyponormal and $p$-paranormal operators, etc..

This note consists of four sections. In Section 2, we introduce some fundamental definitions and properties in the graph theory for our purpose. In Section 3, we obtain some formulae which separates the classes of $p$-hyponormal and $p$-paranormal operators. In Section 4, we provide some new models relevant to weighted directed hand-type trees.

\section{Basic constructions}

In this section we recall basic notions and definitions in [5] or [9]. Let a pair $\mathcal{G}=(V, E)$ be a directed graph if $V \neq \varnothing$ and $E \subset V \times V \backslash\{(v, v): v \in V\}$. An element of $V$ is said to be a vertex of $\mathcal{G}$, a member of $E$ is called an edge of $\mathcal{G}$. Put

$$
\widetilde{E}:=\{\{u, v\} \subseteq V:(u, v) \in E \text { or }(v, u) \in E\} .
$$

A member of $\widetilde{E}$ is called an undirected edge of $\mathcal{G}$. A directed graph $\mathcal{G}$ is called connected if for any two distinct vertices $u$ and $v$ of $\mathcal{G}$, there exists a finite sequence 
$v_{1}, \ldots, v_{n}$ of vertices of $\mathcal{G}$ such that $u=v_{1},\left\{v_{j}, v_{j+1}\right\} \in \widetilde{E}$ for all $j=1, \ldots, n-1$. A finite sequence $\left\{u_{j}\right\}_{j=1}^{n}$ of distinct vertices is said to be a circuit of $\mathcal{G}$ if $n \geq 2$, $\left(u_{j}, u_{j+1}\right) \in E$ for all $j=1, \ldots, n-1$, and $\left(u_{n}, u_{1}\right) \in E$. A vertex $v$ of $\mathcal{G}$ is called a root of $\mathcal{G}$, or briefly $v \in \operatorname{Root}(\mathcal{G})$, if there is no vertex $u$ of $\mathcal{G}$ such that $(u, v)$ is an edge of $\mathcal{G}$. We write $V^{\circ}=V \backslash \operatorname{Root}(\mathcal{G})$. Set

$$
\operatorname{Chi}(u)=\{v \in V:(u, v) \in E\}, \quad u \in V .
$$

Then we call the set $\operatorname{Chi}(u)$, children of $u$ of $\mathcal{G}$. A member of $\operatorname{Chi}(u)$ is called a child of $u$. If for a given vertex $u \in V$, there exists a unique vertex $v \in V$ such that $(v, u) \in E$, then we say that $u$ has a parent $v$ and write $\operatorname{par}(u)$ for $v$. We compose "par" by $k$-times $(k \geq 1)$ and write it by $\operatorname{par}^{k}$. We write $\operatorname{par}^{0}$ for the identity mapping on $V$.

A pair $\mathcal{G}=(V, E, \mu)$ is a weighted directed graph if $(V, E)$ be a directed graph and $\left(V, 2^{V}, \mu\right)$ be a $\sigma$-finite measure space where $2^{V}$ is the power set of $V$. It is wellknown that, since $\left(V, 2^{V}, \mu\right)$ is a $\sigma$-finite measure space, we have that $V$ is at most countable set. A system $\mathfrak{T}=(V, E, \mu)$ is a weighted directed tree if $\mathfrak{T}$ is a weighted directed graph such that $\mathfrak{T}$ is connected, $\mathfrak{T}$ has no circuits, and each vertex $v \in V^{\circ}$ has a parent. In a weighted directed tree $\mathfrak{T}$, we note that $\operatorname{Root}(\mathfrak{T})$ has at most one element. A vertex $v \in V$ is a bifurcated vertex of $\mathfrak{T}$ if it has multi-edges, i.e. the cardinality of children of a bifurcated vertex is greater than one. A vertex $v \in V^{\circ}$ is leaf of $\mathfrak{T}$ if $\operatorname{Chi}(v)=\varnothing$.

Definition 2.1. A weighted directed tree $\mathfrak{T}=(V, E, \mu)$ is hand-type if it has only one bifurcated vertex. In a hand-type directed tree, we write $b$ for the bifurcated vertex.

Definition 2.2. Let $\mathfrak{T}=(V, E, \mu)$ be a weighted directed hand-type tree. The length of trunk of $\mathfrak{T}$ is the number of edges of path from the root to the bifurcated vertex.

Let $\mathfrak{T}=(V, E, \mu)$ be a weighted directed tree. We now consider a measurable transformation $T$ on $V$ defined by

$$
T v= \begin{cases}\operatorname{par}(v) & \text { if } v \in V^{\circ}, \\ \operatorname{root} & \text { if } v=\text { root }\end{cases}
$$

when $\mathfrak{T}$ has a root. If $\mathfrak{T}$ has no root, we define $T v=\operatorname{par}(v)$ for every $v \in V$. If $T$ is non-singular (i.e., $\mu \circ T^{-1} \ll \mu$ ), then $\mu(\operatorname{Chi}(u))=0$ for $u \in V$ with $\mu(\{u\})=0$. Recall that $l^{2}(V)$ is the set of sequences $\left\{\alpha_{v}\right\}_{v \in V}$ such that $\sum_{v \in V}\left|\alpha_{v}\right|^{2}<\infty$. If we define $C_{\mathfrak{T}}: l^{2}(V) \rightarrow l^{2}(V)$ by $C_{\mathfrak{T}}(f)=f \circ T$, then $C_{\mathfrak{T}}$ is bounded if and only if

$$
\sup _{v \in V}|\mu(\operatorname{Chi}(v)) / \mu(\{v\})|<\infty .
$$

Such bounded operator $C_{\mathfrak{T}}$ is called the composition operator induced by a weighted directed tree $\mathfrak{T}$. 


\section{Main characterizations}

Before giving our results, we briefly review some essential properties about the composition operator $C$ on $L^{2}$ ([1] and [2]).

P1. $C$ is normal if and only if $T^{-1} \mathcal{F}=\mathcal{F}$ and $h=h \circ T$.

P2. $C$ is quasinormal if and only if $h=h \circ T$.

P3. $C$ is $p$-hyponormal if and only if $h>0$ and $E\left(1 / h^{p}\right) \leq 1 /\left(h^{p} \circ T\right)$.

P4. $C$ is $p$-paranormal if and only if $E\left(h^{p}\right) \geq h^{p} \circ T$.

We characterize composition operators $C_{\mathfrak{T}}$ induced by a weighted directed handtype tree $\mathfrak{T}=(V, E, \mu)$. Throughout in this paper, we denote a point mass $m(k)$ for the measure $\mu(\{k\})$ and assume that each $m(k)>0$.

Proposition 3.1. Let $\mathfrak{T}=(V, E, \mu)$ be a weighted directed hand-type tree with root $r$ and let $C_{\mathfrak{T}}$ be a composition operator induced by the given $\mathfrak{T}$. Then the composition operator $C_{\mathfrak{T}}$ is quasinormal if and only if the tree $\mathfrak{T}$ has no leaves and the following conditions are hold.

(i) For a vertex $k \in \mathrm{Chi}(r)$, we have that

$$
\frac{\mu(\{r\} \cup \operatorname{Chi}(r))}{m(r)}=\frac{\mu(\operatorname{Chi}(k))}{m(k)} .
$$

(ii) For a vertex $k \in V^{\circ} \backslash \operatorname{Chi}(r)$, we have that

$$
\frac{\mu(\operatorname{Chi}(k))}{m(k)}=\frac{\mu(\operatorname{Chi}(\operatorname{par}(k)))}{m(\operatorname{par}(k))} .
$$

In particular, if the tree $\mathfrak{T}$ has no root, then $C_{\mathfrak{T}}$ is quasinormal if and only if the tree $\mathfrak{T}$ without leaves satisfies the condition (ii).

Proof. For the given hand-type tree $\mathfrak{T}$, if $\mathfrak{T}$ has a leaf $w$ satisfying $\operatorname{Chi}(w)=\varnothing$, then

$$
h(w)=\frac{\mu(\operatorname{Chi}(w))}{m(w)}=0 \text { and } h \circ T(w)=\frac{\mu(\operatorname{Chi}(\operatorname{par}(w)))}{m(\operatorname{par}(w))} .
$$

For each point masses $m(v)>0$ for all $v \in V$, we have that $h(w) \neq h \circ T(w)$. Hence the weighted directed tree $\mathfrak{T}$ has no leaves.

If the tree $\mathfrak{T}$ has the root $r$, then it follows from the definition of transformation $T$ that $T(r)=r$ and $T^{-1}(r)=\{r\} \cup \operatorname{Chi}(r)$. So we obtain that

$$
h(r)=\frac{\mu(\{r\} \cup \operatorname{Chi}(r))}{m(r)} \text { and } h \circ T(r)=h(r) .
$$

Also, for $k \in \operatorname{Chi}(r)$, we have that

$$
h(k)=\frac{\mu(\operatorname{Chi}(k))}{m(k)} \text { and } h \circ T(k)=h(r) .
$$


Finally, for any vertex $k \in V^{\circ} \backslash \mathrm{Chi}(r)$, the following result holds

$$
h(k)=h \circ T(k) \Leftrightarrow \frac{\mu(\operatorname{Chi}(k))}{m(k))}=\frac{\mu(\operatorname{Chi}(\operatorname{par}(k)))}{m(\operatorname{par}(k))} .
$$

Using the above facts and property $\mathrm{P} 2$, we obtain the results for the quasinormality of $C_{\mathfrak{T}}$. Therefore the proof is complete.

Proposition 3.2. Let $\mathfrak{T}=(V, E, \mu)$ be a weighted directed hand-type tree with leaves and let $C_{\mathfrak{T}}$ be a composition operator induced by the given $\mathfrak{T}$.

(i) If a vertex $w$ is a leaf of $\mathfrak{T}$, then $b=\operatorname{par}^{j}(w)$ for some integer $j \geq 1$ and the bifurcated vertex $b$.

(ii) If the tree has a leaf for some integer $j \geq 2$ in (i), then the composition operator induced by the tree has neither p-hyponormality nor p-paranormality.

Proof. By the connectedness of directed hand-type tree, leaves of tree does not appear in forefather generations of the bifurcated vertex $b$ of $\mathfrak{T}$. So leaves of $\mathfrak{T}$ appear on some children generations of the bifurcated vertex $b$. Hence $b=\operatorname{par}^{j}(w)$ for some integer $j \geq 1$ and a leaf $w$ in $\mathfrak{T}$.

Let us set the composition operator $C_{\mathfrak{T}}$ induced by the tree $\mathfrak{T}$. Suppose that a leaf $w$ satisfies $b=\operatorname{par}^{j}(w)$ for some integer $j>1$. From the definition of leaf, we have the Radon-Nikodym derivative function 0 , and so the conditional expectation becomes to the same value at the leaf. But the Radon-Nikodym derivative function at the parent of each leaf is not 0 . Due to the properties P3 and P4, we see that $C_{\mathfrak{T}}$ has neither $p$-hyponormality nor $p$-paranormality.

By the Proposition 3.2, we obtain that if a weighted directed tree with leaves has the $p$-hyponormality (or $p$-paranormality), its leaves are contained in children of the bifurcated vertex.

Theorem 3.3. Let $\mathfrak{T}=(V, E, \mu)$ be a weighted directed hand-type tree and have no root. Let $C_{\mathfrak{T}}$ be a composition operator induced by the given $\mathfrak{T}$. Then we have the following assertions.

(i) $C_{\mathfrak{T}}$ is p-hyponormal if and only if the following two conditions hold;

(i-a) if a vertex $k$ belongs to $\mathrm{Chi}(b)$, then

$$
\sum_{k \in \operatorname{Chi}(b) \backslash\{\text { leaves }\}} \frac{m^{p+1}(k)}{\mu^{p}(\operatorname{Chi}(k))} \leq \frac{m^{p}(b)}{\mu^{p-1}(\operatorname{Chi}(b))} ;
$$

(i-b) $m(k)^{2} \leq m(\operatorname{par}(k)) \cdot \mu(\operatorname{Chi}(k))$ otherwise.

(ii) $C_{\mathfrak{T}}$ is p-paranormal if and only if the following two conditions hold;

(ii-a) if a vertex $k$ belongs to $\mathrm{Chi}(b)$, then

$$
\sum_{k \in \operatorname{Chi}(b) \backslash\{\text { leaves }\}} \frac{\mu^{p}(\operatorname{Chi}(k))}{m^{p-1}(k)} \geq \frac{\mu^{p+1}(\operatorname{Chi}(b))}{m^{p}(b)} ;
$$

(ii-b) $m(k)^{2} \leq m(\operatorname{par}(k)) \cdot \mu(\operatorname{Chi}(k))$ otherwise. 
Proof. (i) We note that the sub- $\sigma$-algebra of a hand-type weighted directed tree $\mathfrak{T}$ is generated by atoms, $\operatorname{Chi}(b)$ and $\cup\{v: \operatorname{par}(v) \neq b\}$. If a vertex $w$ is a leaf of $\mathfrak{T}$, then $h(w)=0$. To find the condition for $p$-hyponormality of composition operator $C_{\mathfrak{T}}$, we need the condition $h>0$. So we may consider a vertex which is not leaf of $\mathfrak{T}$. For the bifurcated vertex $b$, if a vertex $k$ belongs to $\operatorname{Chi}(b)$, then $\operatorname{par}(k)=b$. So we have the followings:

$$
\begin{aligned}
E\left(\frac{1}{h^{p}}\right)(k) & =\frac{1}{\mu(\operatorname{Chi}(b))} \int_{\operatorname{Chi}(b)} \frac{1}{h^{p}(v)} d \mu \\
& =\frac{1}{\mu(\operatorname{Chi}(b))} \sum_{v \in \operatorname{Chi}(b) \backslash\{\text { leaves }\}} \frac{m^{p+1}(v)}{\mu^{p}(\operatorname{Chi}(v))}
\end{aligned}
$$

and

$$
\frac{1}{h^{p} \circ T}(k)=\left(\frac{m(\operatorname{par}(k))}{\mu(\operatorname{Chi}(\operatorname{par}(b)))}\right)^{p}=\left(\frac{m(b)}{\mu(\operatorname{Chi}(b))}\right)^{p}
$$

for any $k \in \operatorname{Chi}(b)$. Otherwise, we obtain that

$$
\operatorname{Chi}(\operatorname{par}(k))=k \text { and } h(k)=\frac{\mu(\operatorname{Chi}(k))}{m(k)} .
$$

So

$$
E\left(\frac{1}{h^{p}}\right)(k)=\frac{1}{m(k)} \int_{\{k\}} \frac{1}{h^{p}(v)} d \mu=\frac{1}{h^{p}(k)}
$$

and

$$
\frac{1}{h^{p} \circ T}(k)=\left(\frac{m(\operatorname{par}(k))}{\mu(\operatorname{Chi}(\operatorname{par}(k)))}\right)^{p}=\left(\frac{m(\operatorname{par}(k))}{m(k)}\right)^{p} .
$$

Therefore for any vertex $k \in V$, we obtain that

$$
\begin{aligned}
E\left(\frac{1}{h^{p}}\right)(k) \leq & \frac{1}{h^{p} \circ T}(k) \Longleftrightarrow \\
& \left\{\begin{array}{l}
\sum_{k \in \operatorname{Chi}(b) \backslash\{\operatorname{leaves}\}} \frac{m^{p+1}(k)}{\mu^{p}(\operatorname{Chi}(k))} \leq \frac{m^{p}(b)}{\mu^{p-1}(\operatorname{Chi}(b))} \\
m(k)^{2} \leq m(\operatorname{par}(k)) \cdot \mu(\operatorname{Chi}(k)) \text { otherwise. }
\end{array}\right.
\end{aligned}
$$

(ii) To prove the paranormality of $C_{\mathfrak{T}}$, we use similar calculations in (i). If a vertex $k \in \operatorname{Chi}(b)$, then we have $\operatorname{par}(k)=b$,

$$
E\left(h^{p}\right)(k)=\frac{1}{\mu(\operatorname{Chi}(b))} \sum_{v \in \operatorname{Chi}(b) \backslash\{\text { leaves }\}} h^{p}(v) \cdot m(v)
$$

and

$$
h^{p} \circ T(k)=h^{p}(\operatorname{par}(k))=\left(\frac{\mu(\operatorname{Chi}(b))}{m(b)}\right)^{p} .
$$


Next, otherwise, we have that the followings:

$$
E\left(h^{p}\right)(k)=\frac{1}{m(k)} \int_{\{k\}} h^{p}(v) d \mu=\left(\frac{\mu(\operatorname{Chi}(k))}{m(k)}\right)^{p}
$$

and

$$
h^{p} \circ T(k)=\left(\frac{\mu(\operatorname{Chi}(\operatorname{par}(k)))}{m(\operatorname{par}(k))}\right)^{p}=\left(\frac{m(k)}{m(\operatorname{par}(k))}\right)^{p} .
$$

So we have that

$$
\begin{aligned}
E\left(h^{p}\right)(k) \geq & h^{p} \circ T(k) \Longleftrightarrow \\
& \left\{\begin{array}{l}
\sum_{k \in \operatorname{Chi}(b) \backslash\{\text { leaves }\}} \frac{\mu^{p}(\mathrm{Chi}(k))}{m^{p-1}(k)} \geq \frac{\mu^{p+1}(\mathrm{Chi}(b))}{m^{p}(b)} \text { if } k \in \operatorname{Chi}(b), \\
m(k)^{2} \leq m(\operatorname{par}(k)) \cdot \mu(\operatorname{Chi}(k)) \text { otherwise. }
\end{array}\right.
\end{aligned}
$$

Hence the proof is complete.

Recall that a weighted directed tree may have at most one root. Now we discuss the $p$-hyponormality and $p$-paranormality of a composition operator for the case of a tree with root.

Theorem 3.4. Let $\mathfrak{T}=(V, E, \mu)$ be a weighted directed hand-type tree with root $r$ and let $C_{\mathfrak{T}}$ be a composition operator induced by the given $\mathfrak{T}$. Then the following assertions hold.

(i) $C_{\mathfrak{T}}$ is p-hyponormal if and only if the following three conditions hold;

(i-a) if a vertex $k$ is root or $k \in \mathrm{Chi}(r)$, it holds that

$$
\sum_{k \in \operatorname{Chi}(r)} \frac{m^{p+1}(v)}{\mu^{p}(\operatorname{Chi}(v))} \leq \frac{m^{p}(r) \cdot \mu(\operatorname{Chi}(r))}{\mu^{p}(\{r\} \cup \operatorname{Chi}(r))} ;
$$

(i-b) if a vertex $k$ belongs to $\mathrm{Chi}(b)$, it holds that

$$
\sum_{k \in \operatorname{Chi}(b) \backslash\{\text { leaves }\}} \frac{m^{p+1}(k)}{\mu^{p}(\operatorname{Chi}(k))} \leq \frac{m^{p}(b)}{\mu^{p-1}(\operatorname{Chi}(b))} ;
$$

(i-c) $m(k)^{2} \leq m(\operatorname{par}(k)) \cdot \mu(\mathrm{Chi}(k))$ otherwise.

(ii) $C_{\mathfrak{T}}$ is p-paranormal if and only if the following three conditions hold;

(ii-a) if a vertex $k$ is root or $k \in \operatorname{Chi}(r)$, it holds that

$$
\sum_{k \in \operatorname{Chi}(r)} \frac{\mu^{p}(\operatorname{Chi}(k))}{m^{p-1}(k)} \geq\left(\frac{\mu(\{r\} \cup \operatorname{Chi}(r))}{m(r)}\right)^{p} \mu(\operatorname{Chi}(r)) ;
$$

(ii-b) if a vertex $k$ belongs to $\mathrm{Chi}(b)$, it holds that

$$
\sum_{k \in \operatorname{Chi}(b) \backslash\{\text { leaves }\}} \frac{\mu^{p}(\operatorname{Chi}(k))}{m^{p-1}(k)} \geq \frac{\mu^{p+1}(\operatorname{Chi}(b))}{m^{p}(b)}
$$


(ii-c) $m(k)^{2} \leq m(\operatorname{par}(k)) \cdot \mu(\operatorname{Chi}(k))$ otherwise.

Proof. To prove our theorem, we first consider two cases of vertex $v$, namely $v$ is either the root $r$ or an element of $\operatorname{Chi}(r)$. Then we combine this result with the statements of Theorem 3.3.

For the given hand-type tree $\mathfrak{T}$ with the root, by the definition of transformation $T$, we have $T(r)=r$ and $T^{-1}(r)=\{r\} \cup \operatorname{Chi}(r)$. So the set $\{r\} \cup \operatorname{Chi}(r)$ is an atom of the sub- $\sigma$-algebra for the $\sigma$-algebra $2^{V}$. Now for a vertex $k \in\{r\} \cup \operatorname{Chi}(r)$, we obtain the followings:

$$
\begin{aligned}
E\left(\frac{1}{h^{p}}\right)(k) & =\frac{1}{\mu(\{r\} \cup \operatorname{Chi}(r))} \int_{\{r\} \cup \operatorname{Chi}(r)} \frac{1}{h^{p}(v)} d \mu \\
& =\frac{1}{\mu(\{r\} \cup \operatorname{Chi}(r))}\left(\sum_{v \in\{r\} \cup \operatorname{Chi}(r)} \frac{m(v)}{h^{p}(v)}\right) \\
& =\frac{1}{\mu(\{r\} \cup \operatorname{Chi}(r))}\left(\frac{m^{p+1}(r)}{\mu^{p}(\{r\} \cup \operatorname{Chi}(r))}+\sum_{v \in \operatorname{Chi}(r)} \frac{m^{p+1}(v)}{\mu^{p}(\operatorname{Chi}(v))}\right) .
\end{aligned}
$$

Hence we can obtain the $p$-hyponormality of $C_{\mathfrak{T}}$ in the cases of $k=r$ or any $k \in \operatorname{Chi}(r)$ :

$$
E\left(\frac{1}{h^{p}}\right)(k) \leq \frac{1}{h^{p} \circ T}(k) \Longleftrightarrow \sum_{v \in \operatorname{Chi}(r)} \frac{m^{p+1}(v)}{\mu^{p}(\operatorname{Chi}(v))} \leq \frac{m^{p}(r) \cdot \mu(\operatorname{Chi}(r))}{\mu^{p}(\{r\} \cup \operatorname{Chi}(r))}
$$

Next, for the $p$-paranormality of $C_{\mathfrak{T}}$ at any vertex $k$ in $\{r\} \cup \operatorname{Chi}(r)$, we have the following:

$$
\begin{aligned}
E\left(h^{p}\right)(k) & =\frac{1}{\mu(\{r\} \cup \operatorname{Chi}(r))} \int_{\{r\} \cup \operatorname{Chi}(r)} h^{p}(v) d \mu \\
& =\frac{1}{\mu(\{r\} \cup \operatorname{Chi}(r))}\left(\frac{\mu^{p}(\{r\} \cup \operatorname{Chi}(r))}{m^{p-1}(r)}+\sum_{v \in \operatorname{Chi}(r)} \frac{\mu^{p}(\operatorname{Chi}(v))}{m^{p-1}(v)}\right) .
\end{aligned}
$$

Therefore we get the $p$-paranormality for $C_{\mathfrak{T}}$ at any vertex $k$ in $\{r\} \cup \operatorname{Chi}(r)$;

$$
\begin{aligned}
E\left(h^{p}\right)(k) & \geq h^{p} \circ T(k) \\
& \Longleftrightarrow \sum_{v \in \operatorname{Chi}(r)} \frac{\mu^{p}(\operatorname{Chi}(v))}{m^{p-1}(v)} \geq\left(\frac{\mu(\{r\} \cup \operatorname{Chi}(r))}{m(r)}\right)^{p} \mu(\operatorname{Chi}(r)) .
\end{aligned}
$$

Finally, for other cases, we join the results in Theorem 3.3. Hence the proof is complete.

Corollary 3.5. Let $\mathfrak{T}=(V, E, \mu)$ be a weighted directed hand-type tree with root $r$ 
and length $\ell$ of trunk of $\mathfrak{T}$. Then the p-hyponormality and p-paranormality for a composition operator $C_{\mathfrak{T}}$, induced by $\mathfrak{T}$, are independent to the length of trunk of T.

Proof. It follows from the Theorem 3.4 that the $p$-hyponormality and $p$ paranormality of $C_{\mathfrak{T}}$ are independent of length $\ell$ of trunk of $\mathfrak{T}$.

Remark 3.6. Let $\mathfrak{T}=(V, E, \mu)$ be a weighted directed hand-type tree and let $C_{\mathfrak{T}}$ be the composition operator induced by the given $\mathfrak{T}$. By the definition of the transformation $T$, we have that $T^{-1}(b)=\operatorname{Chi}(b)$ for the bifurcated vertex $b$ or $T^{-1}$ (root) $=\{$ root $\} \cup$ Chi(root) for the case of $\mathfrak{T}$ with root. From these facts, we see that the sub- $\sigma$-algebra $T^{-1}(\mathcal{F})$ is not equal to the $\sigma$-algebra $\mathcal{F}:=2^{V}$ i.e. the property $\mathrm{P} 1$ does not hold. So we do not have the relation about the normality for the composition operator $C_{\mathfrak{T}}$.

\section{Examples}

Given a directed hand-type tree $\mathfrak{T}=(V, E)$ with the bifurcated vertex $b$. Set $\operatorname{Chi}(b)=\left\{v_{1}, v_{2}, \ldots, v_{n}\right\}$ for some $n \in \mathbb{N}$. Now we consider a point mass measure on $V$ as follows: for any $v \in V$,

$$
m(v)= \begin{cases}x_{i}^{j} & \text { if } v_{i}=\operatorname{par}^{j}(v) \text { for some } j \geq 0 \\ 1 & \text { otherwise }\end{cases}
$$

with $x_{i}>0$ for $i=1, \cdots, n$. Then $\left(V, 2^{V}, m\right)$ is a $\sigma$-finite measure space. And we define a measurable non-singular transformation $T$ on $V$ as the same method in Section 2 .

Firstly, we introduce the familiar example which is considered as model distinguishing partial normalities or weak hyponormalities in [1], [2], or [8].

Example 4.1. We consider the following directed hand-type tree $\mathfrak{T}_{1}$ with no leaves as in Figure 4.1:

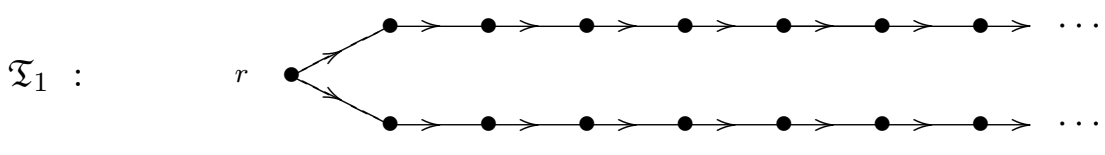

Figure 4.1

We denote the composition operator $C_{\mathfrak{T}_{1}}$ induced by the above tree $\mathfrak{T}_{1}$ as the same process previous. For this tree, the root is equal to the bifurcated vertex $b$. Now we can easily see that $m(v)^{2} \leq m(\operatorname{par}(v)) m(\operatorname{Chi}(v))$ for all $v \in V^{\circ} \backslash \operatorname{Chi}(r)$. Hence, if $v$ is root or $v \in \operatorname{Chi}(r)$, then we have the following results:

$$
C_{\mathfrak{T}_{1}} \text { is quasinormal } \Longleftrightarrow x_{1}=3 \text { and } x_{2}=3,
$$




$$
C_{\mathfrak{T}_{1}} \text { is } p \text {-hyponormal } \Longleftrightarrow\left(\frac{3}{x_{1}}\right)^{p}+\left(\frac{3}{x_{2}}\right)^{p} \leq 2
$$

and

$$
C_{\mathfrak{T}_{1}} \text { is } p \text {-paranormal } \Longleftrightarrow\left(\frac{x_{1}}{3}\right)^{p}+\left(\frac{x_{2}}{3}\right)^{p} \geq 2 .
$$

This results are the just same as in [1] and [2].

Next, we show a directed tree with leaf in the next two examples.

Example 4.2. Consider the following directed hand-type tree $\mathfrak{T}_{2}$ without root as in Figure 4.2: We denote the composition operator $C_{\mathfrak{T}_{2}}$ induced by the above

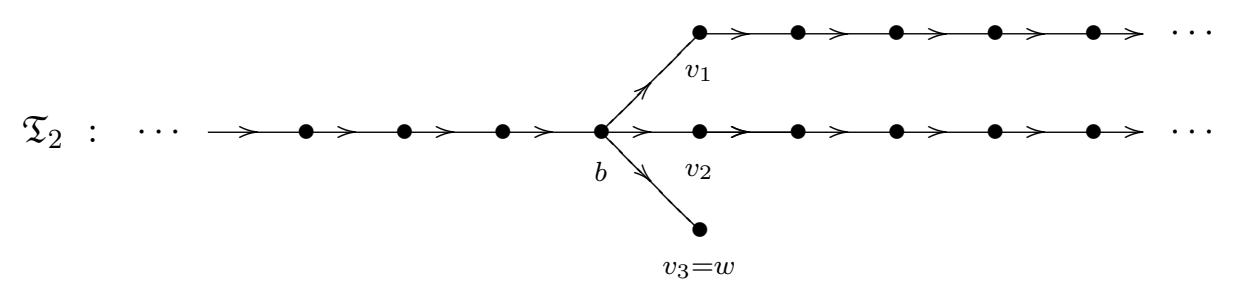

Figure 4.2

tree $\mathfrak{T}_{2}$ as the same process previous. It follows from the construction of tree $\mathfrak{T}_{2}$ that $\mathfrak{T}_{2}$ has no root but contains one leaf. By simple calculations, we have $m(v)^{2} \leq m(\operatorname{par}(v)) m(\operatorname{Chi}(v))$ for all $v \in V \backslash \operatorname{Chi}(b)$. Therefore we have the following results: for $v \in \operatorname{Chi}(b)$,

$$
C_{\mathfrak{T}_{2}} \text { is } p \text {-hyponormal } \Longleftrightarrow\left(\frac{2}{x_{1}}\right)^{p}+\left(\frac{2}{x_{2}}\right)^{p} \leq 2
$$

and

$$
C_{\mathfrak{T}_{2}} \text { is } p \text {-paranormal } \Longleftrightarrow x_{1}{ }^{p}+x_{2}{ }^{p} \geq 2^{p+1} .
$$

Finally we introduce a hand-type tree $\mathfrak{T}_{3}$ having root and leaves.

Example 4.3. Consider the another directed hand-type tree $\mathfrak{T}_{3}$ with root $r$ and say the length $\ell=4$ as in Figure 4.3: Now we modify the point mass measure in above defined as follows:

$m(r):=1, m(u):=2^{j-1}$ for $r=\operatorname{par}^{j}(u), u \in V(j=1,2,3,4)$ and for other vertex $v$ in $V^{\circ}$,

$$
m(v):= \begin{cases}x_{i}^{j} & \text { if } v_{i}=\operatorname{par}^{j}(v) \text { for some } j \geq 1 \\ m(b) & \text { otherwise }\end{cases}
$$

with $x_{i}>0$ for $i=1,2,4$. Then we can obtain the composition operator $C_{\mathfrak{T}_{3}}$ induced by the weighted directed tree $\mathfrak{T}_{3}$ as the same process previous. From the modified point mass measure in above, we can easily have that $m(v)^{2} \leq$ 


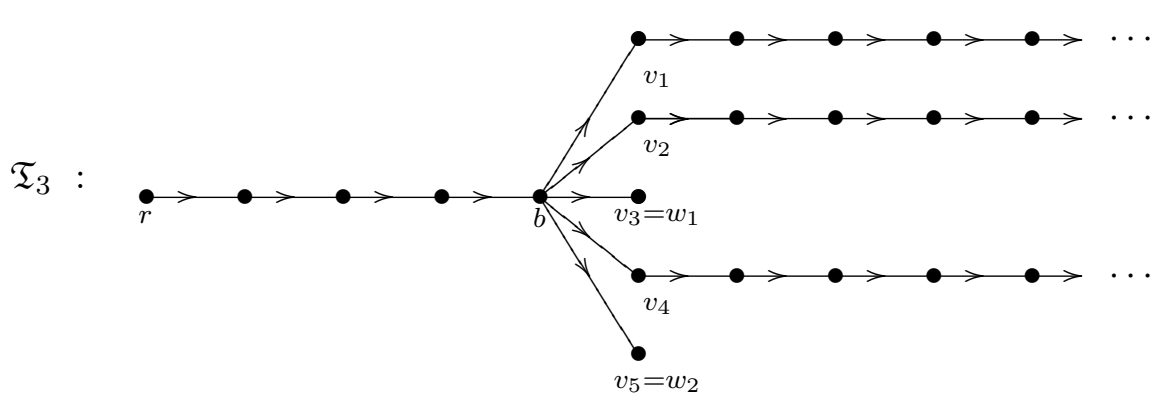

Figure 4.3

$m(\operatorname{par}(v)) m(\operatorname{Chi}(v))$ for all $v \in V^{\circ} \backslash \operatorname{Chi}(b)$. Finally, if $v$ is root, $v \in \operatorname{Chi}(r)$, or $v \in \operatorname{Chi}(b)$, then we have the following assertions:

$$
C_{\mathfrak{T}_{3}} \text { is } p \text {-hyponormal } \Longleftrightarrow\left(\frac{24}{x_{1}}\right)^{p}+\left(\frac{24}{x_{2}}\right)^{p}+\left(\frac{24}{x_{4}}\right)^{p} \leq 3
$$

and

$$
C_{\mathfrak{T}_{3}} \text { is } p \text {-paranormal } \Longleftrightarrow x_{1}{ }^{p}+x_{2}{ }^{p}+x_{4}{ }^{p} \geq 3 \cdot 24^{p} .
$$

\section{References}

[1] C. Burnap and I. Jung, Composition operators with weak hyponormality, J. Math. Anal. Appl., 337(2008), 686-694.

[2] C. Burnap, I. Jung and A. Lambert, Separating partial normality classes with composition operators, J. Operator Theory, 53(2005), 381-397.

[3] G. Exner, I. Jung and M. R. Lee, Block matrix operators and weak hyponormalities, Integr. equ. theory, 65(2009), 345-362.

[4] T. Furuta, Invitation to linear operators, Taylor \& Francis Inc., 2001.

[5] Z. J. Jabłoński, I. B. Jung and J. Stochel, On weighted shifts on directed trees, submitted

[6] I. B. Jung, M. R. Lee and P. S. Lim, Gaps of operators, II, Glasgow Math. J., 47(2005), 461-469.

[7] I. Jung, M. R. Lee and S. Park, Separating classes of composition operators via subnormal condition, Proc. Amer. Math. Soc., 135(2007), 3955-3965.

[8] M. R. Lee and J. W. Park, Weakly hyponormal composition operators and Embry condition, Kyungpook Math. J., 49(2009), 683-689. 
[9] O. Ore, Theory of graphs, Amer. Math. Soc. Coll. Publ. Vol. XXXVIII, 1962.

[10] M. Rao, Conditional measures and Applications, Marcel Decker, New York 1993.

[11] D. Xia, Spectral theory of hyponormal operators, Birkhauser Verlag, Basel · Boston - Stuttgart, 1983. 